\title{
Leçon tirée d'une expérience de consentement au don d'organes provenant de donneurs décédés
}

\author{
Sam D. Shemie MDCM
}

Citation : CMAJ 2021 November 15;193:E1735-6. doi : 10.1503/cmaj.190761-f

Voir la version anglaise de l'article ici : www.cmaj.ca/lookup/doi/10.1503/cmaj.190761; voir l'article connexe au www.cmaj.ca/lookup/ doi/10.1503/cmaj.210836-f

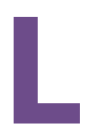

e système de dons et de transplantations d'organes au Canada subit actuellement une transformation qui le fait passer d'un état fragmentaire et peu performant ${ }^{1}$, à un régime de collaboration qui s'améliore constamment (https:// www.cihi.ca/fr/rapport-electronique-sur-les-statistiques-relatives -aux-transplantations-aux-listes-dattente-et-aux). Un donneur potentiel entre en contact avec le système de santé selon une trajectoire prévisible, soit : soins préhospitaliers, service des urgences, unité de soins intensifs et bloc opératoire. Tant pour les familles que pour les professionnels de la santé, les étapes au cours desquelles on tente de sauver une vie, on se résout à accepter une mort inéluctable, puis on amorce un don d'organes forment un processus complexe, délicat et éprouvant du point de vue émotionnel, même s'il est prévisible. Une optimisation de chacune de ces étapes est nécessaire pour améliorer le système. Les organismes provinciaux responsables des dons d'organes portent la charge opérationnelle de la gestion du processus de don et du lien avec les receveurs d'une transplantation. Dans un article de recherche connexe, Singh et ses collègues ont évalué les facteurs qui influent sur le consentement au don d'organes en Ontario ${ }^{2}$, une province qui a mis en œuvre bon nombre des éléments fondamentaux de l'amélioration du système pour augmenter le nombre de dons d'organes provenant de donneurs décédés. Les conclusions des auteurs apportent des réponses à certaines questions, mais en soulèvent d'autres à la lumière de récents changements apportés à la politique sur les dons d'organes en Nouvelle-Écosse.

Même s'il reste beaucoup à faire pour réduire les taux de mortalité et d'invalidité chez les patients en attente d'une transplantation, les efforts concertés et coordonnés entre les hôpitaux, les programmes de transplantations, les professionnels et les sociétés qui regroupent les intensivistes et les urgentologues se sont améliorés. Le système de dons après le décès a profondément changé depuis ses tout débuts alors qu'on faisait face à un manque de connaissances, à de la résistance, de l'inconfort et une perception de conflit d'intérêts chez les familles des donneurs; les soins de fin de vie intègrent désormais la culture du don d'organes provenant après le décès. Ce changement repose sur la

\section{Points clés}

- L'amélioration du rendement des systèmes de dons d'organes passe par la mise en œuvre de directives nationales inspirées par la communauté pour gérer les dons potentiels à chaque étape du processus : maladie ou traumatisme, décès, prélèvement d'organe et transplantation.

- Une fois les pratiques de base bien en place, le prochain défi majeur consiste à faire augmenter les taux de consentement.

- Le Canada tire de l'arrière par rapport aux autres pays pour ce qui est de la collecte des données sur le rendement en matière de dons d'organes qui peuvent être utiles pour orienter les politiques.

- Dans les systèmes où le consentement doit être donné, on peut améliorer les taux de consentement en s'attaquant à des facteurs modifiables tels que la formation du personnel, la participation des médecins aux discussions sur le consentement, l'enregistrement du consentement et l'atténuation de la préséance des volontés de la famille sur celles des personnes décédées.

- Le récent changement législatif en Nouvelle-Écosse pour mettre en place un consentement présumé a créé une expérience sociale qui permettra aux chercheurs d'observer si la politique peut améliorer les taux de dons d'organes sans compromettre le droit légitime des individus de refuser de faire don de leurs organes.

création et la mise en œuvre de pratiques exemplaires nationales inspirées de la communauté et appuyées par les sociétés professionnelles, et de lignes directrices pour chaque étape du processus, appuyées par Santé Canada et coordonnées par la Société canadienne du sang (et son prédécesseur, le Conseil canadien pour le don et la transplantation). Il existe à présent des directives concernant la détermination de la mort cérébrale ${ }^{3}$, la prise en charge des donneurs ${ }^{4,5}$, le don après la détermination de la mort cardiocirculatoire ${ }^{6}$ et après la réanimation à la suite d'un arrêt cardiaque ${ }^{7}$, l'identification des donneurs et l'acheminement rapide des demandes (après vérification des donneurs potentiels) ${ }^{8}$, les discussions sur le consentement au don chez les personnes en fin de vie ${ }^{9}$, les dons d'organes à la suite d'une aide 
médicale à mourir ${ }^{10}$ et la formation du personnel des unités de soins concernées ${ }^{11}$. Le rendement actuellement très variable des systèmes provinciaux de dons d'organes et de transplantations pourrait dépendre de l'importance que chacun accorde à ces pratiques fondamentales et à leur mise en œuvre.

Une fois qu'un système de dons a optimisé les pratiques de bases, le dernier défi pour améliorer les taux de dons d'organes et de transplantations est de miser davantage sur le consentement. Le rendement de l'Ontario est semblable à celui du Royaume-Uni où les taux de dons d'organes et de transplantations ont augmenté substantiellement, avec des améliorations minimes aux taux de consentement (www.odt.nhs.uk/). Même si les patients conscients, par exemple, ceux qui demandent l'aide médicale à mourir $^{10}$, peuvent offrir leur propre consentement éclairé, ce sont habituellement des mandataires, présumément au fait des volontés ou des préférences des patients si ceux-ci sont comateux ou agonisants, qui fournissent le consentement.

Les mentalités ont changé dans les hôpitaux au sujet du don d'organes, mais la société n'a pas toujours suivi, comme en témoignent les taux de consentement. Le taux de consentement global reste à $60 \%$ et contraste avec le taux de soutien de $88 \%$ à $95 \%$ exprimé par le public en faveur du don d'organes après le décès (https://profedu.blood.ca/sites/msi/files/2020_ipsos_public_ opinion_polling_-_approval_of_organ_donation_after_death.pdf). Les facteurs modifiables qui pourraient jouer sur les taux de consentement incluent approche individualisée par du personnel dûment formé, participation du médecin aux discussions sur le consentement, enregistrement préalable du consentement et préséance du choix des familles sur les volontés des personnes décédées. Les auteurs d'un article de recherche connexe ont identifié ces facteurs modifiables, mais on ignore si leur modification peut influer sur les taux de consentement. Le Canada tire de l'arrière par rapport à d'autres pays pour ce qui est de recueillir des statistiques sur les dons qui pourraient fournir des renseignements sur le rendement, y compris des données qui permettraient le calcul de taux de consentement comparatifs. La préséance du choix des familles sur les volontés exprimées par les personnes décédées demeure un défi et rappelle l'écart entre la loi et la pratique. En vertu de la loi canadienne, les familles n'ont aucune autorité légale pour refuser un don si la personne décédée a fourni un consentement valide ${ }^{12}$, et pourtant la préséance de la volonté des familles sur un consentement enregistré et légalement valide pourrait être un important facteur modifiable. On comprend le personnel des unités de soins intensifs et des programmes de dons d'organes de ne pas vouloir entrer en conflit avec les familles lorsque les patients sont en fin de vie et il ignore parfois qu'il a l'autorité légale pour que soient respectées les volontés de la personne décédée plutôt que celles de sa famille. Aux États-Unis, ce problème a été résolu par l'adoption de "l'autorisation de procéder » au don d'organes, en vertu d'un mandat légal par lequel les volontés du donneur sont respectées et non celles de la famille ${ }^{13}$.

Pour faire face à la pénurie d'organes et pour combler l'écart entre le soutien exprimé par le public et les taux réels observés dans la pratique, on a souvent lancé des appels à modifier le système de consentement dans les médias de masse et chez les politiciens afin de transformer le refus présumé en consentement présumé. Les pays dotés d'un système de consentement présumé ont tendance à avoir des taux de dons et de transplantations plus élevés. Même si le consentement présumé vise à améliorer les taux de dons et de transplantations d'organes, le paramètre le plus pertinent demeure son incidence sur les taux de consentement; or, ce paramètre est omis dans plusieurs rapports publiés. Les données du pays de Galles ont montré des améliorations statistiquement significatives des taux de dons et de consentements depuis la mise en place d'un système de consentement présumé ${ }^{14}$. En mai 2020, l'Angleterre a adopté une nouvelle loi sur le consentement présumé (https://www.organdonation.nhs.uk/ uk-laws/organ-donation-law-in-england/). En janvier 2021, la Nouvelle-Écosse est devenue le premier territoire de compétence en Amérique du Nord à adopter un système de consentement présumé (https://novascotia.ca/organ-and-tissue-donation -changes/fr/).

Le consentement présumé n'affectera pas substantiellement les taux de dons et de transplantations d'organes à moins que les systèmes de santé ne s'assurent également que les hôpitaux sont aptes à les gérer en appliquant des pratiques exemplaires. Ces dernières incluent des processus pour permettre les dons après un décès cardiaque, l'identification et l'orientation rapides et obligatoires des donneurs potentiels, le maintien d'un personnel spécialisé en dons d'organes, la capacité clinique entourant les services de dons et de transplantations et des données complètes pour mesurer le rendement. La Nouvelle-Écosse a reconnu la nécessité d'investir dans la consolidation du système au moment de l'adoption du consentement présumé.

Étant donné ces exigences, de nombreuses questions demeurent quant à la meilleure façon d'optimiser le consentement. Par exemple, un système provincial devrait-il s'attaquer aux variables modifiables du système de refus présumé, comme l'indiquent les auteurs de la recherche connexe? Ou le système devrait-il plutôt travailler activement à l'adoption du consentement présumé, en tenant compte que ce dernier suscitera aussi une amélioration des taux de références de donneurs? La société peut-elle améliorer les taux de consentements et de dons sans compromettre le droit légitime au refus de faire un don d'organe? Quelle est la meilleure stratégie pour améliorer les taux de consentements dans une société multiethnique? Les responsables des orientations politiques, les responsables des systèmes de dons et de transplantations d'organes, et les universitaires observeront étroitement l'incidence sociale de la nouvelle politique néo-écossaise. Même si les caractéristiques démographiques ethnoculturelles varient d'une province à l'autre, il sera intéressant pour le pays entier de comparer les expériences de consentement présumé de la Nouvelle-Écosse et de refus présumé en Ontario.

\section{Références}

1. Gill JS, Klarenbach S, Cole E, et al. Deceased organ donation in Canada: an opportunity to heal a fractured system. Am J Transplant 2008;8:1580-7.

2. Singh JM, Ball IM, Hartwick M, et al. Factors associated with consent for organ donation: a retrospective population-based study. CMAJ 2019;191:E1725-32.

3. Shemie SD, Doig C, Dickens B, et al.; Pediatric Reference Group; Neonatal Reference Group. Severe brain injury to neurological determination of death: Canadian forum recommendations. CMAJ 2006;174:S1-13. 
4. Shemie SD, Ross H, Pagliarello J, et al.; Pediatric Recommendations Group. Organ donor management in Canada: recommendations of the forum on Medical Management to Optimize Donor Organ Potential. CMAJ 2006;174:S13-32.

5. Ball IM, Hornby L, Rochwerg B, et al. Management of the neurologically deceased organ donor: a Canadian clinical practice guideline. CMAJ 2020;192:E361-9.

6. Shemie SD, Baker AJ, Knoll G, et al. Donation after cardiocirculatory death in Canada. CMAJ 2006;175(Suppl):S1-24.

7. Grunau B, Hornby L, Singal RK, et al. Extracorporeal cardiopulmonary resuscitation for refractory out-of-hospital cardiac arrest: the state of the evidence and framework for application. Can J Cardiol 2018;34:146-55.

8. Zavalkoff S, Shemie SD, Grimshaw J, et al.; Potential Organ Donation Identification and System Accountability (PODrosoph Inf ServA) Conference Participants (Appendix). Potential organ donor identification and system accountability: expert guidance from a Canadian consensus conference. Can J Anaesth 2019;66:432-47.

9. Shemie SD, Robertson A, Beitel J, et al.; EOL Conversations with Families of Potential Donors participants. End-of-life conversations with families of potential donors: leading practices in offering the opportunity for organ donation. Transplantation 2017;101(Suppl 1):S17-26
10. Downar J, Shemie SD, Gillrie C, et al.; Canadian Blood Services, the Canadian Critical Care Society, the Canadian Society of Transplantation and the Canadian Association of Critical Care Nurses. Deceased organ and tissue donation after medical assistance in dying and other conscious and competent donors: guidance for policy [erratum publié dans CMAJ 2019;191:E745]. CMAJ 2019;191:E604-13.

11. Shemie SD, MacDonald S.; Canadian Blood Services - Canadian Critical Care Society Expert Consultation Group. Improving the process of deceased organ and tissue donation: a role for donation physicians as specialists. CMAJ 2014; 186:95-6.

12. Toews M, Caulfield T. Evaluating the "family veto" of consent for organ donation. CMAJ 2016;188:E436-7.

13. Stahler PA, Weese SE, Nygaard RM, et al. Honoring patients' organ donation decisions when family conflict is present: experience from a single organ procurement organization. J Trauma Acute Care Surg 2014;77:555-8.

14. Madden S, Collett D, Walton $\mathrm{P}$, et al. The effect on consent rates for deceased organ donation in Wales after the introduction of an opt-out system. Anaesthesia 2020;75:1146-52.
Intérêts concurrents : Sam Shemie est conseiller médical contractuel pour la Société canadienne du sang. Aucun autre intérêt concurrent n'a été déclaré.

Cet article a été sollicité et il n'a pas été révisé par des pairs.

Affiliations : Division de médecine de soins intensifs, Hôpital de Montréal pour enfants, Centre universitaire de santé McGill; Université McGill; Institut de recherche du CUSM, Montréal, Qc; Programme Don provenant de personnes décédées, Société canadienne du sang, Ottawa, Ont.
Propriété intellectuelle du contenu : Il s'agit d'un article en libre accès distribué conformément aux modalités de la licence Creative Commons Attributions (CC BY-NC-ND 4.0), qui permet l'utilisation, la diffusion et la reproduction dans tout médium à la condition que la publication originale soit adéquatement citée, que l'utilisation se fasse à des fins non commerciales (c.-à-d., recherche ou éducation) et qu'aucune modification ni adaptation n’y soit apportée. Voir : https:// creativecommons.org/licenses/by-nc-nd/4.0/deed.fr.

Correspondance : Sam Shemie, sam.shemie@mcgill.ca 